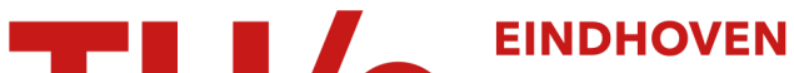 \\ UNIVERSITY OF \\ TECHNOLOGY
}

\section{Comparison of nonlocal approaches in continuum damage mechanics}

Citation for published version (APA):

Vree, de, J. H. P., Brekelmans, W. A. M., \& Gils, van, M. A. J. (1995). Comparison of nonlocal approaches in continuum damage mechanics. Computers and Structures, 55(4), 581-588. https://doi.org/10.1016/00457949(94)00501-S

DOI:

10.1016/0045-7949(94)00501-S

Document status and date:

Published: 01/01/1995

Document Version:

Publisher's PDF, also known as Version of Record (includes final page, issue and volume numbers)

Please check the document version of this publication:

- A submitted manuscript is the version of the article upon submission and before peer-review. There can be important differences between the submitted version and the official published version of record. People interested in the research are advised to contact the author for the final version of the publication, or visit the $\mathrm{DOI}$ to the publisher's website.

- The final author version and the galley proof are versions of the publication after peer review.

- The final published version features the final layout of the paper including the volume, issue and page numbers.

Link to publication

\section{General rights}

Copyright and moral rights for the publications made accessible in the public portal are retained by the authors and/or other copyright owners and it is a condition of accessing publications that users recognise and abide by the legal requirements associated with these rights.

- Users may download and print one copy of any publication from the public portal for the purpose of private study or research.

- You may not further distribute the material or use it for any profit-making activity or commercial gain

- You may freely distribute the URL identifying the publication in the public portal.

If the publication is distributed under the terms of Article $25 \mathrm{fa}$ of the Dutch Copyright Act, indicated by the "Taverne" license above, please follow below link for the End User Agreement:

www.tue.nl/taverne

Take down policy

If you believe that this document breaches copyright please contact us at:

openaccess@tue.nl

providing details and we will investigate your claim. 


\title{
COMPARISON OF NONLOCAL APPROACHES IN CONTINUUM DAMAGE MECHANICS
}

\author{
J. H. P. de Vree, W. A. M. Brekelmans and M. A. J. van Gils \\ Faculty of Mechanical Engineering, University of Technology, P.O. Box 513, 5600 MB Eindhoven. \\ The Netherlands
}

(Received 4 December [993)

\begin{abstract}
A local continuum damage theory and two distinct nonlocal variants are applied to model the failure behaviour of a construction made of macroscopically brittle material. In the nonlocal formulations a material characteristic length parameter is introduced associated with the width of the microstructural damage zone. The numerical implementation of the approaches has been performed in a finite element code. Simulation results calculated for a plane stress configuration are compared. The local approach solutions show severe lack of mesh objectivity, whereas both the nonlocal solutions converged after nesh refinement. By adequate tuning of the nonlocal descriptions mutually similar responses can be obtained. although intrinsic differences are present in the resulting damage distributions.
\end{abstract}

\section{INTRODUCTION}

Continuum damage approaches offer the possibility to simulate the mechanical behaviour of constructions of history-dependent material, irreversibly degenerating under mechanical loads. The damage process is characterized by the development, growth and coalescence of microdefects leading to the formation and propagation of macrocracks and eventually to rupture. Microstructural observations are useful for the identification and quantification of history parameters associated with damage [1]. The introduction of damage parameters is adopted from Kachanov [2], who first applied the damage concept to model fracture in creep. Although damage parameters are conceptually related to the observable local deterioration of the material, generally these parameters are phenomenologically incorporated into the constitutive description. In this paper, brittle material behaviour will be considered: elastic moduli are influenced by damage development. The damage evolution is assumed to be isotropic; explicit time dependence and thermal effects are excluded from the considerations.

The increase of damage generally leads to local softening behaviour: the tangential stiffness becomes negative. It has been shown $[3,4]$ that a local approach to softening phenomena may lead to a physically unacceptable localization of the deformation; in a finite element context serious mesh sensitivity occurs [5-7]. To ovcrcome the deficiencies of the classical local modelling a number of basically different approaches can be distinguished:

- nonlocal modelling of the constitutive behaviour $[4,8,9]$;

- gradient dependent material description $[4,10]$;
- micropolar (Cosserat) continuum theory [4];

- viscous regularization [1], 12];

- local manipulation of material properties depending on the element size [11].

Gradient dependent models as well as micropolar continuum theory require radical modifications of ordinary finite element codes. It is obvious that viscous regularization is inappropriate for typically brittle material behaviour. The adaptation of material properties is disputable from a physical point of view: global responses may reasonably be predicted, however, calculated local strain and damage distributions will certainly not be in agreement with reality. In this paper two different nonlocal theories are examined and the major structure of the finite element implementation will be explained. The numerical results of a characteristic example are reported and discussed in conclusion.

\section{THE LOCAL DAMAGE APPROACH}

\subsection{General concepts}

The influence of isotropic damage on elastic material behaviour is described by the so-called damage parameter $D$. This monotonously increasing scalar quantity, $0 \leqslant D \leqslant 1$, expresses the level of material degradation [13-16]. Undamaged material is characterized by $D=0$, the complete loss of stiffness and coherence by $D=1$. According to the classical continuum damage mechanics approach the preposition of the principle of effective stress $[13,14]$ leads to the constitutive relationship

$$
\sigma=(1-D) \mathbf{H} \epsilon
$$


with $\sigma$ the column with Cauchy stress components, $\epsilon$ the column with strain components and with $\mathbf{H}$ Hooke's elasticity matrix. The product $(1-D) \mathbf{H}$ can be considered as the effective elasticity matrix. Implicitly it is assumed that Poisson's ratio $v$ is not affected by damage.

The damage evolution is determined by a scalar measure of the strain components: the damage equivalent strain $\epsilon_{\mathrm{eq}}=\epsilon_{\mathrm{eq}}(\epsilon) \geqslant 0$. There are several alternatives $[17,18]$ to define $\epsilon_{c q}$, weighting the strain components differently. A suitable selection has to be made in accordance with material features. In this paper a modification (adopted from Ref. [19]) of the Von Mises equivalent strain will be applied:

$$
\begin{aligned}
& \epsilon_{\mathrm{eq}}=\frac{\gamma-1}{2 \gamma(1-2 v)} J_{1} \\
&+\frac{1}{2 \gamma} \sqrt{\left(\frac{\gamma-1}{1-2 v} J_{1}\right)^{2}+\frac{12 \gamma}{(1+v)^{2}} J_{2}^{\prime}}
\end{aligned}
$$

with $\gamma$ denoting the ratio of tensile and compressive strength and with $J_{1}$ and $J_{2}^{\prime}$ strain invariants defined by

$$
\begin{aligned}
J_{1}= & \epsilon_{x x}+\epsilon_{y y}+\epsilon_{z z} \\
J_{2}^{\prime}= & \frac{1}{3}\left(\epsilon_{x x}^{2}+\epsilon_{y y}^{2}+\epsilon_{z z}^{2}-\epsilon_{x x} \epsilon_{y y}-\epsilon_{y y} \epsilon_{z=}\right. \\
& \left.-\epsilon_{z z} \epsilon_{x x}+3\left(\epsilon_{x y}^{2}+\epsilon_{y z}^{2}+\epsilon_{z x}^{2}\right)\right) .
\end{aligned}
$$

The subscripts $x, y, z$ in eqn (3) indicate the (strain) components in an orthonormal co-ordinate system. Increase of damage is only possible if actually $\epsilon_{\mathrm{eq}}$ equals an evolving threshold value $\kappa \geqslant 0$ and increases. The damage remains constant if $\epsilon_{\mathrm{eq}}$ is smaller than $\kappa$ or if $\epsilon_{\mathrm{eq}}$ is not increasing. These statements are mathematically formulated by

$$
\begin{array}{lll}
\dot{D}<0 & \text { if } \quad \epsilon_{\mathrm{e} \varphi}=\kappa & \text { and if } \dot{\epsilon}_{\mathrm{e} 4}>0 \\
\dot{D}=0 & \text { if } \quad \epsilon_{\mathrm{e} 4}<\kappa & \text { or if } \quad \dot{\epsilon}_{\mathrm{eq}} \leqslant 0 .
\end{array}
$$

Consistency requires that

$$
\begin{aligned}
& \dot{\kappa}=\left\langle\dot{\epsilon}_{\mathrm{eq}}\right\rangle \quad \text { if } \quad \epsilon_{\mathrm{eq}}=\kappa \\
& \dot{\kappa}=0 \text { if } \quad \epsilon_{\mathrm{cq}}<\kappa
\end{aligned}
$$

where the so-called McAuley brackets $\langle\cdot\rangle$ are defined by

$$
\langle x\rangle=\frac{1}{2}(x+|x|) .
$$

The nondecreasing threshold strain quantity $\kappa$ is comparable with the yield stress in the theory of elastoplasticity. Strain tensors satisfying $\epsilon_{\mathrm{t} y}=\kappa$ represent the actual damage surface in strain space.
For strain tensors mapped within this surface the damage rate is zero, while for increasing damage the strain tensors should be mapped on the damage surface. The damage development for brittle materials is governed by a damage evolution law which relates the value of the actual damage $D$ to the actual threshold parameter $\kappa$. In Section 4 a particular specification of $D=D(\kappa)$ will be used for further elaborations.

\subsection{Aspects of the finite element implementation}

An arbitrary structural problem is described by the equilibrium requirements, constitutive relationships and boundary conditions. This general problem formulation usually only admits a solution by approximation. The finite element method is applied to generate the system of discretized nodal equilibrium equations. The nonlinearity, a consequence of the incorporation of damage, necessitates an incremental iterative solution procedure. The most recent estimate for the column with nodal displacements ${ }^{k} u_{i+1}$ in increment $k$, iteration $i$, is determined from

$$
{ }^{k} u_{i+1}={ }^{k} u_{i}+{ }^{k} \Delta u_{i}={ }^{k} u_{i}+\left({ }^{k} \mathbf{K}_{i}\right){ }^{k}{ }^{k}
$$

with ${ }^{k} \mathbf{K}_{i}$ the global tangential stiffness matrix and ${ }^{k} r_{i}$ the column with residual forces, resulting from the previous iteration step. To calculate the matrix ${ }^{k} \mathbf{K}_{i}$ the local tangential stiffness matrix

$$
{ }^{k} \mathbf{S}_{i}=\left(\frac{\mathrm{d} \sigma}{\mathrm{d} \epsilon}\right)_{t=h_{i}}
$$

should be evaluated for the actual iterative approximation at every intergration point. Based on the constitutive modelling in Section 2.1 the matrix ${ }^{k} \mathbf{S}_{i}$ can be decomposed into

$$
{ }^{k} \mathbf{S}_{i}={ }^{k} \mathbf{V}_{i}+{ }^{k} \mathbf{W}_{i}
$$

with ${ }^{k} \mathbf{V}_{i}$ the elastic and ${ }^{k} \mathbf{W}_{i}$ the softening contribution, respectively,

$$
{ }^{k} \mathbf{V}_{i}=\left(1-{ }^{k} D_{1}\right) \mathbf{H}, \quad{ }^{k} \mathbf{W}_{i}=-\frac{{ }^{k} \sigma_{i}}{1-{ }^{k} D_{i}}\left(\frac{\mathrm{d} D}{\mathrm{~d} \epsilon}\right)_{i=k_{i}}
$$

To achieve a consistent iteration scheme the matrix ${ }^{k} \mathbf{W}_{i}$ should be determined according to the following elaboration:

$$
\begin{aligned}
& { }^{k} \mathbf{W}_{i}=-\frac{{ }^{k} \sigma_{i}}{1-{ }^{k} D_{i}}\left(\frac{\mathrm{d} D}{\mathrm{~d} k}\right)_{i=h_{k i}}
\end{aligned}
$$

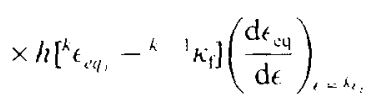


with $\left.h^{[}\right]$the Heaviside function defined by

$$
h[x]= \begin{cases}0 & \text { if } x \leqslant 0 \\ 1 & \text { if } x>0\end{cases}
$$

and with ${ }^{k} \kappa_{i}$ the actual threshold value given by

$$
{ }^{k} \kappa_{i}=\max \left[{ }^{k} \epsilon_{\mathrm{eq},}, \quad{ }^{k-1} \kappa_{\mathrm{f}}\right]
$$

where the subscript $f$ refers to the final converged state in the previous increment $(k-1)$. It is remarked that for ${ }^{k} \mathbf{S}_{i}$ an almost zero matrix is substituted when ${ }^{k} \kappa_{i}$ approaches the critical value $\kappa_{c}$ associated with $D\left(\kappa_{c}\right)=1$, to avoid numerical problems.

The column with residual forces ${ }^{k} r_{i}$ in the right hand side of eqn (7) is equal to the difference of the external load ${ }^{k} f_{\text {ext }}$ and the internal forces ${ }^{k} f_{\text {int, }}$ to be determined from the actual stress state ${ }^{k} \sigma_{i}$ *

$$
{ }^{k} r_{i}={ }^{k} f_{\mathrm{ext}}-{ }^{k} f_{\text {int }}\left({ }^{k} \sigma_{i}\right) \text {. }
$$

The iteration process is terminated when the above unbalance becomes negligibly small.

\section{NONLOCAL DAMAGE FORMULATIONS}

\subsection{General concepts}

Two different nonlocal formulations are considered with respect to the applicability in brittle fracture simulations, particularly the regularization of deformation localization. Both procedures will be explained in detail in Sections 3.3 and 3.4. Preliminary to that the common features are presented.

The essential idea of the nonlocal approach is the negligence of the generally acceptcd principlc of local action, stating that, in the absence of temperature effects, the stress in a material point is completely determined by the deformation and the deformation history at that point. The actual internal damage parameter $D$ figuring in the constitutive eqn (1) is now assumed to be dependent on the strain (and the strain history) in a limited finite area enclosing the particular material point where the stress has to be evaluated. Consequently local strain peaks will always have a certain transfer to the environment and thus prevent the localization of the damage, which in turn has a suppressing effect on the progressive growth of the deformation. The nonlocal constitutive relationship for an arbitrary material point reads:

$$
\sigma=(1-\bar{D}) \mathbf{H} \epsilon
$$

where $\bar{D}$ depends on the distribution of the strain and the strain history in the vicinity of the material point. In a formal expression the quantity $\bar{D}$ at time $t$ in a point $x$ in the surrounding volume $V(x)$ can be written as:

$$
\bar{D}(x, t)=\bar{D}\{\epsilon(\xi, \tau) \mid \xi \in V(x) ; \tau \leqslant t\} .
$$

Analogous to eqn (4) the nonlocal damage quantity $\bar{D}$ is assumed to be expressible in a weighted version $\bar{\epsilon}_{\mathrm{eq}}$ of the equivalent strain:

$$
\begin{array}{llll}
\dot{\bar{D}}>0 & \text { if } \quad \bar{\epsilon}_{\mathrm{eq}}=\kappa & \text { and if } \quad \dot{\bar{\epsilon}}_{\mathrm{eq}}>0 \\
\dot{\bar{D}}=0 & \text { if } \quad \bar{\epsilon}_{\mathrm{eq}}<\kappa & \text { or if } \quad \dot{\bar{\epsilon}}_{\mathrm{eq}} \leqslant 0,
\end{array}
$$

while the threshold $\kappa$ satisties

$$
\begin{aligned}
& \dot{\kappa}=\left\langle\dot{\bar{\epsilon}}_{\mathrm{eq}}\right\rangle \quad \text { if } \quad \bar{\epsilon}_{\mathrm{eq}}=\kappa \\
& \dot{\kappa}=0 \quad \text { if } \quad \bar{\epsilon}_{\mathrm{eq}}<\kappa
\end{aligned}
$$

to maintain a consistent formulation.

\subsection{Aspects of the finite element implementation}

In a finite element context the weighted damage $\bar{D}^{P}$ in a particular integration point $P$ can be calculated from the nonlocal equivalent strain $\bar{\epsilon}_{\text {eq }}^{P}$ (and the history) defined by

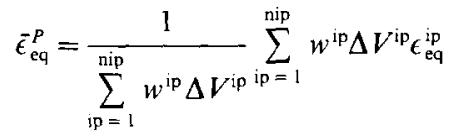

with ip the index of an integration point in a set of $P$-surrounding integration points, nip the total number of integration points in that set, $\Delta V^{\text {ip }}$ the integration volume associated with integration point ip and $w^{\text {ip }}$ the weighting function to be evaluated at the distance $r$ from point $P$ to the integration point. Based on eqn (15), the iterative variation of the stress column, necessary to determine the stiffness matrix, can be formulated as

$$
\begin{aligned}
\delta \sigma & =(1-\bar{D}) \mathbf{H} \delta \epsilon-\delta \bar{D} \mathbf{H} \epsilon \\
& =(1-\bar{D}) \mathbf{H} \delta \epsilon-\delta \bar{D} \frac{\sigma}{1-\bar{D}}
\end{aligned}
$$

while $\delta \bar{D}$ can be expressed in $\delta \bar{\epsilon}_{\mathrm{eq}}$ by

$$
\delta \bar{D}=\frac{\mathrm{d} \bar{D}}{\mathrm{~d} \kappa} \frac{\mathrm{d} \kappa}{\mathrm{d} \bar{\epsilon}_{\mathrm{eq}}} \delta \bar{\epsilon}_{\mathrm{eq}} .
$$

By incorporation of the relationships eqns (18) and (19) the iterative variation of the nonlocal damage in integration point $P$ can be written as

$$
\delta \bar{D}^{\mathrm{P}}=\left(\frac{\mathrm{d} \bar{D}}{\mathrm{~d} \kappa}\right)^{P} h\left[\bar{\epsilon}_{\mathrm{eq}}^{P}-\kappa^{P}\right] h\left[\dot{\bar{\epsilon}}_{\mathrm{cq}}^{P}\right]\left(\sum_{i p=1}^{n i p} \frac{\partial \bar{\epsilon}_{\epsilon \mathrm{q}}^{P}}{\partial \epsilon_{\mathrm{eq}}^{i p}} \frac{\mathrm{d} \epsilon_{\mathrm{cq}}^{i p}}{\mathrm{~d} \epsilon^{i p}} \delta \epsilon^{i p}\right) .
$$

The partial derivative of the nonlocal equivalent strain in point $P$ to the local equivalent strain in point ip reads:

$$
\frac{\delta \epsilon_{\mathrm{eq}}^{p}}{\delta \epsilon_{\mathrm{eq}}^{i p}}=W^{i p} \frac{\Delta V^{i p}}{V^{P}}
$$




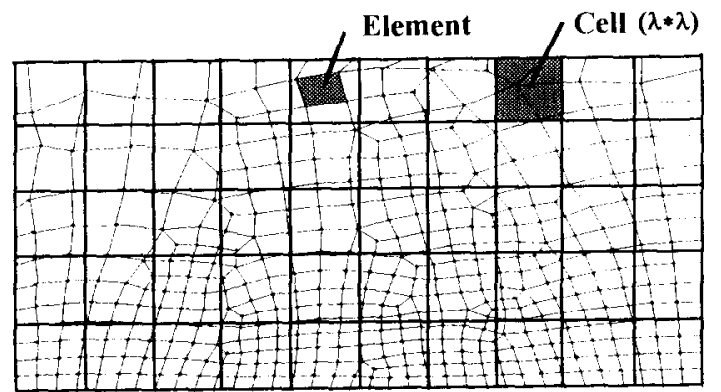

Fig. 1. Elentent mesh and cell grid.

with

$$
V^{p}=\sum_{i p=1}^{n i p} w^{i p} \Delta \boldsymbol{V}^{i p}
$$

In eqn (22) the variation of damage in one integration point $P$ is coupled to the variation of the strain in a certain number ( $n$ ip) of integration points, reflecting the nonlocal character of the modelling procedure. The amount for this interaction in a standard finite element scheme, to achieve a consistent iterative solution strategy, requires severe adaptations in the computer code. Therefore the solution technique has been chosen inconsistently. neglecting the communication between different integration points. as far as the stiffness matrix is concerned. This implies that eqn (22) is replaced by the approximation:

$$
\begin{aligned}
& \dot{\delta} \bar{D}^{P}=\left(\frac{\mathrm{d} \bar{D}}{\mathrm{~d} k}\right)^{P} h\left[\tilde{\epsilon}_{\mathrm{eq}}^{P}-\kappa^{P}\right] h\left[\dot{\bar{\epsilon}}_{\mathrm{eq}}^{P}\right] \cdots(r-0) \\
& \times \frac{\Delta V^{p}}{V^{P}} \frac{\mathrm{d} c^{p}}{\mathrm{~d}^{P}{ }^{P}} \delta_{\epsilon}{ }^{P} .
\end{aligned}
$$

The following stiffness matrix contributions for increment $k$ in iteration step $i$, comparable to the relationships in eqn (10), are found:

$$
\begin{aligned}
& { }^{k} \mathbf{V}_{i}=\left(1-{ }^{k} \bar{D}_{i}\right) \mathbf{H}, \\
& { }^{k} \mathbf{W}_{i}=-\frac{{ }^{k} \sigma_{i}}{1-{ }^{k} \bar{D}}\left(\frac{\partial \bar{D}}{\partial \kappa}\right)_{k=k_{k i}} h\left[{ }^{k} \tilde{\epsilon}_{\mathrm{eq}_{t}}-{ }^{k}{ }^{1} k_{\mathrm{f}}\right] \\
& \times w(r=0) \frac{\Delta V}{V} \frac{\mathrm{d} \epsilon_{\mathrm{eu}}}{\mathrm{d} t},
\end{aligned}
$$

expressed in local quantities only (the superscript $P$. denoting the particular integration point, is omitted in these equations).

\subsection{The grid method}

Among the variety of methods of averaging $[6,20]$, in this section a strategy is pursued inspired by the observation that, at microstructural level, many materials have a characteristic volume where the damage distribution is almost uniform [21]. The dimension of this characteristic volume is related to the size of matcrial inhomogeneities as the constituing particles or aggregates.

The grid method to be presented here, is merely adopted from Hall and Hayhurst [21]. However, it will be applied in a modified manner. A regular cell grid overlaly is placed over an element mesh. Under mesh refinement the grid remains unchanged. The measures of the cells are equal to the characteristic dimension of the material, indicated by the length parameter $\lambda$. In Fig. 1 a grid with typical cells is shown. Essential for the method is the assumption that in every cell of the grid the nonlocal equivalent strain and consequently the nonlocal damage is considered as constant. The local equivalent strains of all individual integration points within the cell are averaged with uniform weighting. The assignment of element integration points to the cells is performed

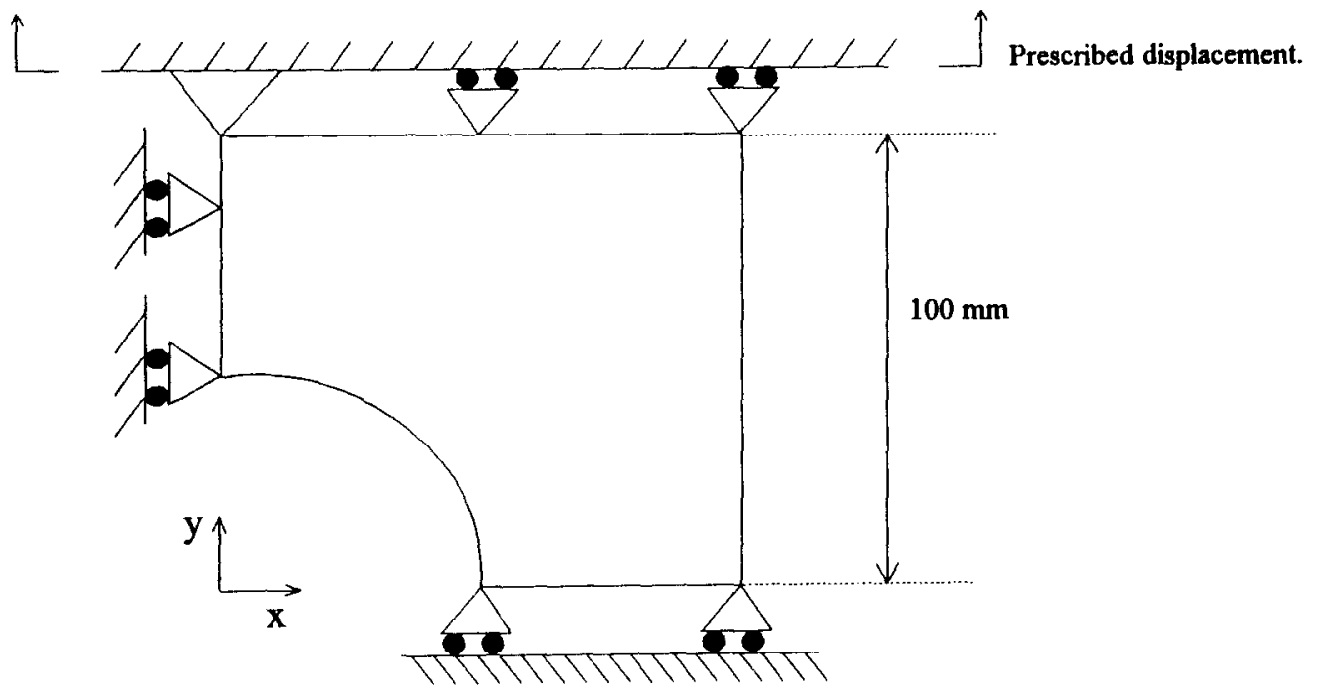

Fig. 2. Configuration and boundary conditions. 


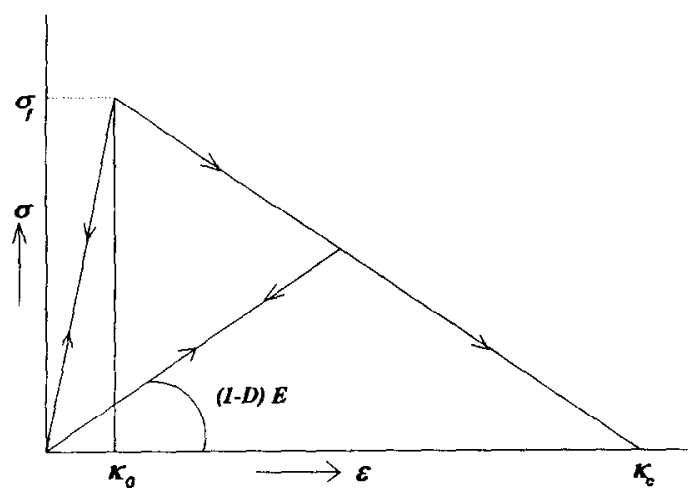

Fig. 3. Stress-strain relationship.

only once at the beginning of the analysis and does not change during load incrementation. As a consequence of the procedure proposed, the calculated nonlocal damage will be discontinuous over the boundaries of adjacent cells.

\subsection{The continuous average strain method}

The nonlocal equivalent strain in an arbitrary integration point $P$ can also be evaluated as the weighted average of equivalent strains in all material points within a certain radius. This method has been described by Bazant ef al. $[8,22]$ and by Saourides and Mazars [9]. This method principally leads to a continuous damage field. The nonlocal equivalent strain in a particular point $P$ is determined with eqn (19) by a summation over the (nip) integration points with distance $r$ to $P$ not exceeding the material characteristic length $\hat{\lambda}$ (with nip a known number, depending on the local size of the elements in the relevant vicinity of point $P$ ), while the weighting function $w$ is supposed to be a function of the distance. For further elaborations $w(r)$ has been selected as a Gaussian weighting function according to Bazant [8]:

$$
w(r)=\mathrm{e}^{-(\boldsymbol{r} / \hat{\lambda})^{2}}
$$

where $\alpha$ is a control parameter. Bazant and PijaudierCabot [8] recommended $\alpha=2$ in the case of two

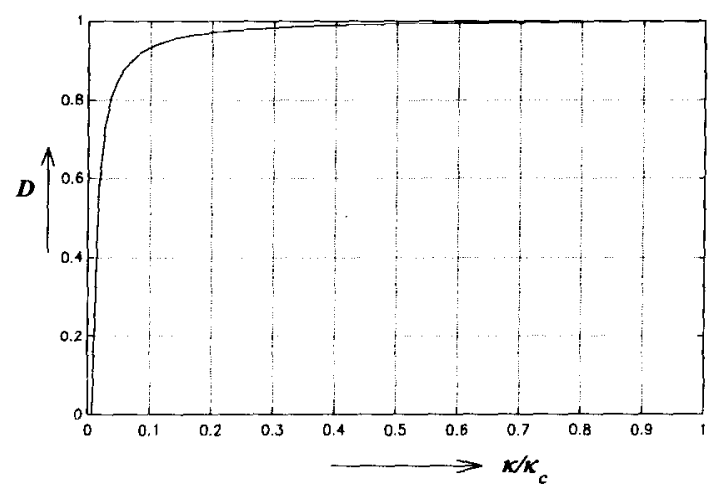

Fig. 4. The damage $D$ as a function of threshold $\kappa / \kappa_{c}$.

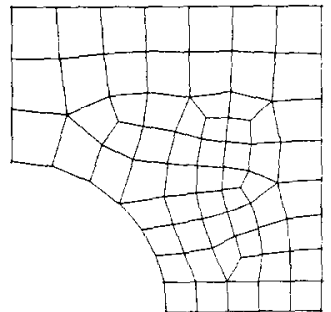

Mesh 1: 63 elements

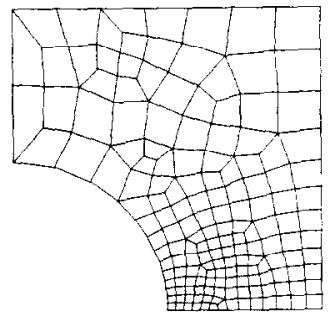

Mesh 3: 163 elements

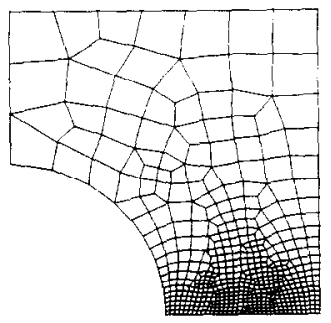

Mesh 5: 504 elements

Fig. 5. Meshes for the quarter plate.

dimensional configurations; however, the background for this proposal is not quite clear.

\section{TEST PROBLEM AND MATERIAL MODEL}

To examine the applicability of the methods out lined above a relatively simple configuration model with an inhomogeneous strain field is considered: a square plate $(200 \times 200 \mathrm{~mm}$, thickness $1 \mathrm{~mm})$ with a central circular hole (radius $50 \mathrm{~mm}$ ). The plate is supported at one side and loaded with a prescribed displacement at the opposite side. Because of symmetry the analysis is performed for the upper right quarter of the plate, using boundary conditions as indicated in Fig. 2. To ensure physical relevance the

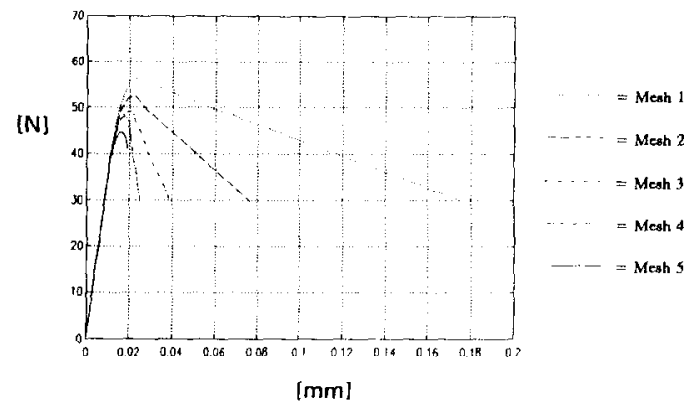

Fig. 6. Force-displacement diagram, local modelling. 


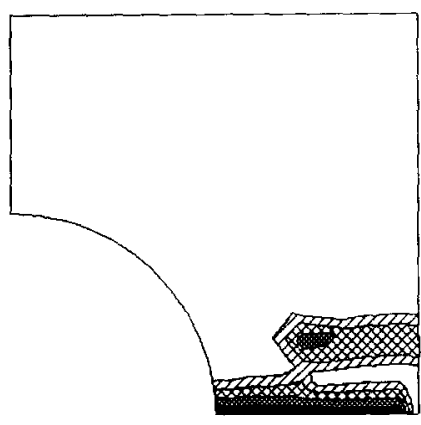

Mesh 2

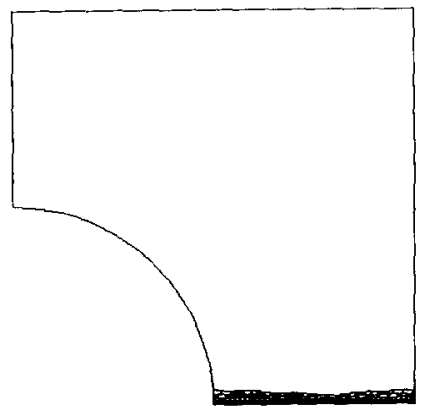

Mesh 5

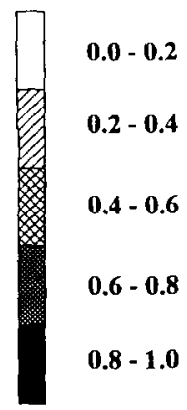

Fig. 7. Damage distribution, local modelling.

material description has been adopted from related research in Ref. [23]. The one dimensional stress-strain relationship (tension) is bilinear as illustrated in Fig. 3. This bilinear material behaviour matches with the local constitutive modelling in Section 2 if $D=D(\kappa)$ is specified by

$$
D(\kappa)=1-\frac{\kappa_{0}}{\kappa} \frac{\left(\kappa_{c}-k\right)}{\left(\kappa_{c}-\kappa_{0}\right)}
$$

with $\kappa_{0}$ and $\kappa_{c}$ the initial and the critical values of $\kappa$, respectively. Equation (28) is visualized in Fig. 4. The material data [23] used for the numerical calculations are: $E=6000 \mathrm{MPa}, v=0.3, \gamma=10, \kappa_{0}=2 \times 10^{-4}$, $\kappa_{\mathrm{c}}=2.7 \times 10^{-2}$ and $i-10 \mathrm{~mm}$. The nonlocal relationship $\bar{D}=\bar{D}(\kappa)$, required for elaboration of the approaches in Section 3 is taken as equivalent to eqn (28).

The quarter of the plate to be analysed is subdivided into isoparametric four-node plane stress elements. In order to study the mesh dependence, five different meshes (Fig. 5) are applied to discretize the displacement field. The element size varies from large to small in the region of the plate where high gradients of the strain and consequently of the damage are to be expected.

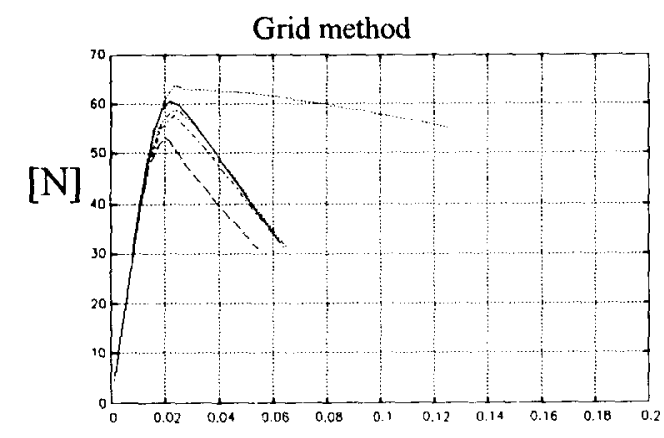

[mm]

\section{RESULTS}

\subsection{Local modelling}

The response results of the analysis with the local damage concept are presented in Fig. 6. The variable along the horizontal axis is the prescribed displacement of the upper edge of the quarter plate model and along the vertical axis the associated total tensile force. Typical differences in the distributions of the damage, determined with a coarse and with a fine mesh, are visualized in Fig. 7 for a tensile force equal to $40 \mathrm{~N}$ in the post-peak softening regime. The objectionable consequence of local modelling is obvious. Mesh refinement leads to a vanishing energy dissipation and the deformation localizes in an area with decreasing volume.

\subsection{Nonlocal modelling}

Figure 8 displays the responses calculated with the grid method and the continuous average strain method using Gaussian weighting with $\alpha=2$, see eqn (27). It can be observed that for both methods the results converge with refinement of the mesh. However, these two nonlocal regularization procedures predict quite different responses. In Figs 9 and 10 the damage distributions are presented, again for a

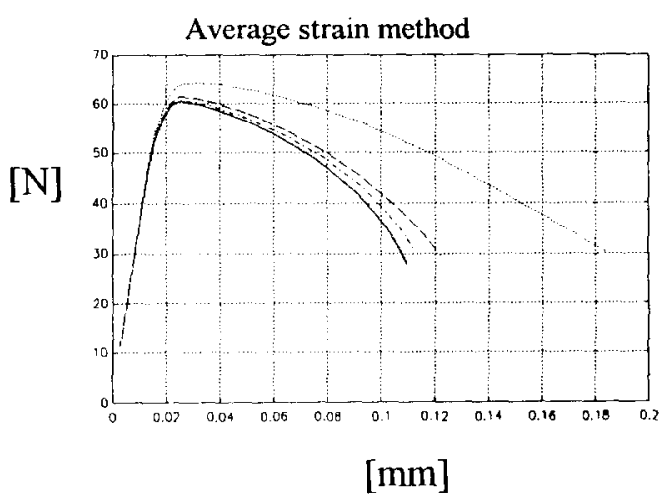

Mesh 2;

Mesh 3;

Mesh 4; Mesh 5;

Fig. 8. Force displacement diagrams, nonlocal modelling. 


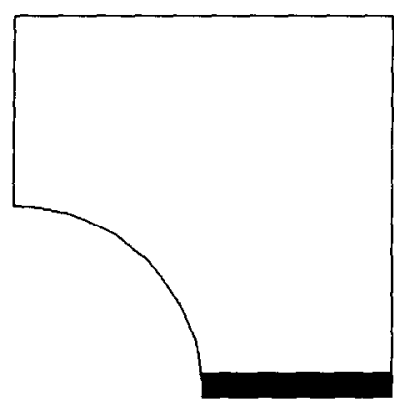

Mesh 2

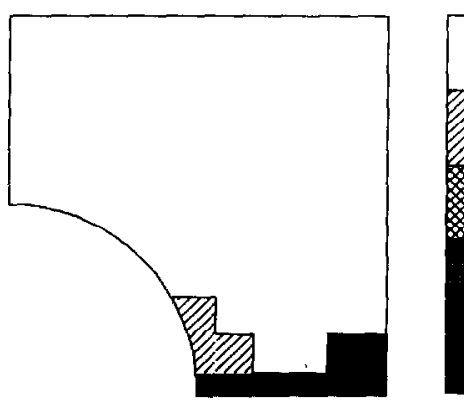

Mesh 5
$0.0-0.2$

$0.2-0.4$

$0.4-0.6$

$0.6-0.8$

$0.8-1.0$

Fig. 9. Damage distribution, grid method.

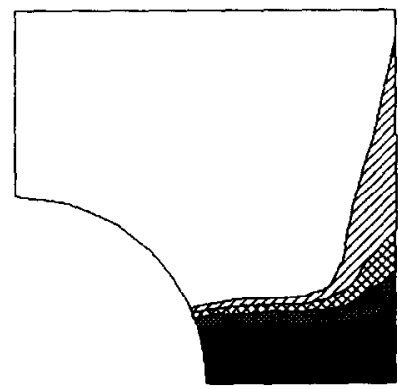

Mesh 2

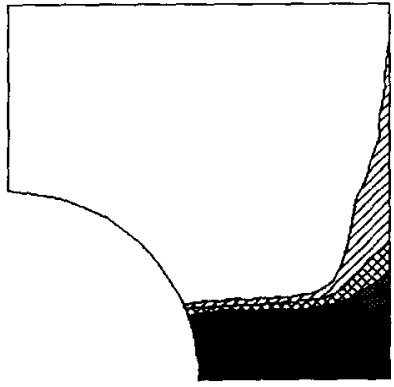

Mesh 5

Fig. 10. Damage distribution, average strain method.

tensile force of $40 \mathrm{~N}$ on the softening path. Especially in Fig. 10 it can be observed that for a course mesh the damage distribution already closely resembles the distribution determined with the finest mesh. Based on the considerations of dissipated energy, the results of the grid method can be accessed as rather satisfying: therefore these results are accepted as a reference and it is examined for which value of the weighting parameter $\alpha$ in eqn (27) the average strain method produces a similar response to the grid method. Figure 11 gives the responses calculated with the average strain method for variations of the parameter $\alpha$. Obviously the responses from the grid method and the average strain method approximately coincide for

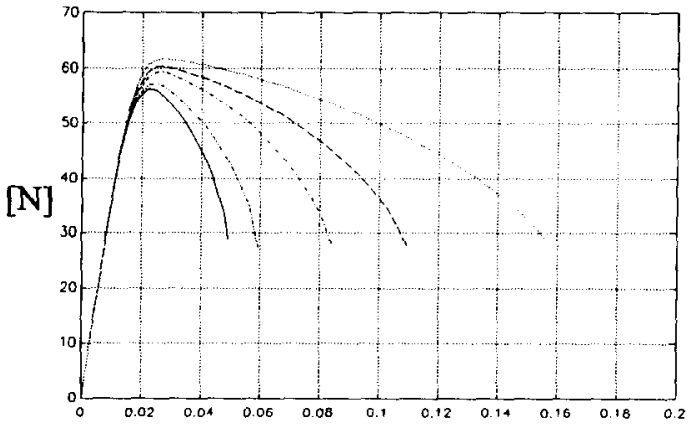

$[\mathrm{mm}]$

$$
\alpha=1 ;-\cdots-\alpha=2 ; \quad \cdots-\alpha=3 ; \quad \alpha=4 ; \quad-\alpha=5 ;
$$

Fig. 11. Force-displacement diagrams for different values of $\alpha$, average strain method. $\alpha=4$. In Fig. 12 the damage distribution resulting from the average strain method for $\alpha=4$ is displayed.

\section{CONCLUSIONS AND DISCUSSION}

Continuum damage theories lead to powerful methods for the numerical analysis of the behaviour of softening materials, provided that adequate precautions are taken to limit the localization of the deformation and consequently of the damage. The problems of the mesh objectivity of finite element solutions and correct representation of size effects are intrinsically related. With the application of distinguishable nonlocal approaches in continuum damage mechanics, mesh objective mesh results were obtained in this paper for brittle material behaviour.

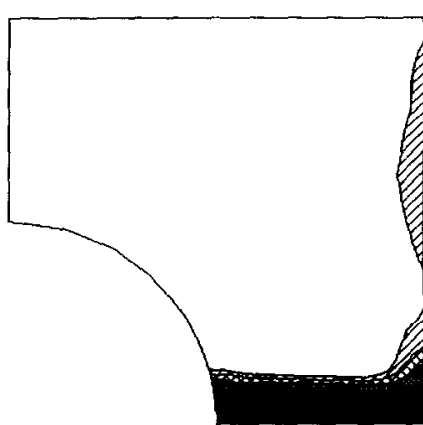

$0.0-0.2$

$0.2-0.4$

$0.4-0.6$

$0.6-0.8$

$0.8-1.0$

Fig. 12. Damage distribution with $\alpha=4$, average strain method. 
For the grid method and the weighted average strain method convergence occurred with mesh refinement. These methods can easily be implemented into a classical finite element computer code; the increase of CPU-time needed for the nonlocal calculations proposed is small. Manipulations in the continuous weighted average strain method to produce appropriate results, leads to a value of approximately 4 for the weighting parameter $\alpha$. This value is not in accordance with the recommendation in the literature [8], originating from mathematical considerations. This deviation, here actually established for a particular example, should be examined in a more general context in the continuation of this research.

Apart from the availability of sufficient material data to describe the local softening phenomena, a number of suitable choices have to be made to achieve a proper simulation of damaging configurations. Using the theory presented in this paper, the following items have to be addressed carefully:

- the definition of the equivalent strain; every material has its own characteristic internal disintegration mechanism associated with some combination of strain components. Therefore a variety of equivalent strain definitions is known in the literature leading to different simulation results. The correct choice to simulate experimental observation can only be accessed by hybrid numerical and experimental research;

- the particular quantity in the nonlocal approach that will be averaged; in this paper averaging of the equivalent strain has been elaborated. In the literature also other choices have been made [21];

- the averaging method; two essentially different averaging procedures have been considered in this paper, leading to a discontinuous and a continuous description of damage evolution. Microstructural investigations should give evidence of the physical relevance of one of these approaches for a particular material. The weighted average strain method offers a number of possibilities for tuning the modelling description on physical experience by adjusting the weighting function [24].

Acknowledgement -The results presented in this paper have been obtained by implementation of the theoretical developments into the DIANA finite element code (DIANA Analysis by, Delft, the Netherlands). The authors gratefully acknowledge D. G. Roddeman (from T.N.O. Building and Construction Research, Delft, The Netherlands) for his support

\section{REFERENCES}

1. W. Becker and D. Gross, A one dimensional micromechanical model of elastic-microplastic damage evolution. Acta Mech. 70, 221-233 (1987).

2. L. M. Kachanov, Time of the rupture process under creep conditions. Tzv. Akad. Nauk. SSR. Otd. Tekh. 8, 26-31 (1958).

3. M. A. Crisfield, Local instabilities in the non linear analysis of reinforced concrete beams and slabs. Proc. Inst. Civil Engrs. 73, 135-145 (1982).

4. R. de Borst and H. B. Mühlhaus, Continuum models for discontinuous media. Proce InI. RILEM/ESIS Conf. on Fracture Processes in Concrete, Rock and Ceramics (Edited by J. G. M. van Mier, J. G. Rots and A. Bakker), pp. 601 618. Noordwijk (1991).

5. S. T. Pietruszczak and Z. Mroz, Finite element analysis of the deformation of strain-softening materials. $I \mathrm{ht} . J$. numer. Meth. Engng 17, 327-334 (1981).

6. Z. P. Bazant, F. B. Lin and G. Pijaudier-Cabot. Yield limit degradation: nonlocal continuum model with local strain. Proc. Int. Conf. on Computational Plasticity (Ediled by E. Onde, R. Owen and E. Hinton), pp. 1757-1779. Barcelona (1987).

7. A. Needleman, Material rate dependence and mesh sensitivity in localization problems Compul. Meth Appl. Mech. Engng 67, 69-85 (1988).

8. Z. P. Bazant and G. Pijaudier-Cabot, Nonlocal continuum damage, localization instability and convergence. J. appl. Mech. 55, 287-293 (1988).

9. C. Saouridis and J. Mazars, A multiscale approach to distributed damage and its usefulness for capturing structural size effects. Proc. France-U.S. Workshop on Strain Localization and Size Effects due to Cracking and Damage (Edited by J. Mazars and Z. P. Bazant), pp. 391-403, Cachan (1988).

10. N. Triantafylidis and E. C. Aifantis, A gradient approach to localization of deformation. I. Hyperelastic materials. J. Elast. 16, 225-237 (1986).

11. J. C. Simo, Strain softening and dissipation: a unification of approaches. Proc. France-U.S. Workshop on Strain Localization and Size Effects due to Cracking and Damage (Editcd by J. Mazars and Z. P. Bazant), pp. 440-461, Cachan (1988).

12. R. de Borst, Gradient-dependent plasticity: formulation and algorithmic aspects. Int. J. numer. Meth. Engng 35 , $521-539(1992)$.

13. J. L. Chaboche, Continuum damage mechanics. Parts 1 and 2. J. appl. Mech. 55, 59-72 (1988).

14. J. C. Simo and J. W. Ju, Strain- and stress-based continuum damage models. Parts 1 and 2. Int. J. Solids Strucl. 23, 821-869 (1987).

15. J. Lemaitre, Local approach of fracture. Engng Fract. Mech. 25, 523-537 (1986).

16. L. M. Kachanov, Introduction to Continuum Damage Mechanics. Martinus Nijhoff, Dordrecht (1986).

17. L. J. M. G. Dortmans and G. de With, Weakest-link failure predictions for ceramics. Part IV: Application of mixed mode fracture criteria for multi-axial loading. J. eur. Ceram. Soc. 10, 109-114 (1992).

18. W. A. M. Brekelmans, P. J. G. Schreurs and J. H. P. de Vree, Continuum damage mechanics for softening of brittle materials. Acta Mech. 93, 133-143 (1992).

19. J. G. Williams, Siress Analysis of Polymers. Longman, London (1973).

20. Z. P. Bazant, Mechanics of distributed cracking. Appl. Mech. Rev. 39, 665-705 (1986).

21. F. R. Hall and D. R. Hayhurst, Modelling of grain size effects in creep crack growth using a nonlocal continuum damage approach. Proc. R. Soc. Lond. A 433, 405-421 (1991).

22. Z. P. Bazant and F. B. Lin, Non-local yield limit degradation. Int. I. numer. Meth. Fngng 26, 1805 1823 (1988).

23. J. Carmeliet and H. Hens, Fracture of a fabric reinforced mortar based on a stochastic approach to initial damage. Proc. Conf. on Localized Damage 11: Fatigue and Fracture Mechanics (Edited by M. H. Aliabadi), pp. 283-298 (1992).

24. Z. P. Bazant and T. P. Chang, Instability of nonlocal continuum and strain averaging. J. Engng Mech. Dir. ASCE 110, 1441 1450(1984). 